\title{
Impacts of the overexpression of a tomato translationally controlled tumor protein (TCTP) in tobacco revealed by phenotypic and transcriptomic analysis
}

\author{
Márcio de Carvalho ${ }^{1}$ Márcio Luís Acencio ${ }^{2,4}$. \\ Alessandra Vasconcellos Nunes Laitz ${ }^{1}$ Laura Migliorini de Araújo ${ }^{1}$. \\ Mariana de Lara Campos Arcuri ${ }^{1} \cdot$ Leandro Costa do Nascimento $^{3} \cdot$ Ivan G. Maia $^{1}$
}

Received: 9 September 2016 / Accepted: 7 February 2017 / Published online: 4 March 2017

(C) Springer-Verlag Berlin Heidelberg 2017

\begin{abstract}
Key message Overexpression of a tomato TCTP impacts plant biomass production and performance under stress. These phenotypic alterations were associated with the up-regulation of genes mainly related to photosynthesis, fatty acid metabolism and water transport.

Abstract The translationally controlled tumor protein (TCTP) is a multifaceted and highly conserved eukaryotic protein. In plants, despite the existence of functional data implicating this protein in cell proliferation and growth, the detailed physiological roles of many plant TCTPs remain poorly understood. Here we focused on a yet uncharacterized TCTP from tomato (SITCTP). We show that, when overexpressed in tobacco, SITCTP may promote plant biomass production and affect performance under salt and
\end{abstract}

Communicated by Marcelo Menossi.

Electronic supplementary material The online version of this article (doi:10.1007/s00299-017-2117-0) contains supplementary material, which is available to authorized users.

Ivan G. Maia

igmaia@ibb.unesp.br

1 Departamento de Genética, Instituto de Biociências, UNESP, Botucatu, SP 18618-970, Brazil

2 Departamento de Física e Biofísica, Instituto de Biociências, UNESP, Botucatu, SP, Brazil

3 Laboratório Central de Tecnologias de Alto Desempenho em Ciências da Vida (LaCTAD), UNICAMP, Campinas, SP, Brazil

4 Department of Cancer Research and Molecular Medicine, Faculty of Medicine, Norwegian University of Science and Technology (NTNU), Trondheim 8905, MH 7491, Norway osmotic stress. Transcriptomic analysis of the transgenic plants revealed the up-regulation of genes mainly related to photosynthesis, fatty acid metabolism and water transport. This induced photosynthetic gene expression was paralleled by an increase in the photosynthetic rate and stomatal conductance of the transgenic plants. Moreover, the transcriptional modulation of genes involved in ABA-mediated regulation of stomatal movement was detected. On the other hand, genes playing a pivotal role in ethylene biosynthesis were found to be down-regulated in the transgenic lines, thus suggesting deregulated ethylene accumulation in these plants. Overall, these results point to a role of TCTP in photosynthesis and hormone signaling.

Keywords Translationally controlled tumor protein · TCTP $\cdot$ Abiotic stress $\cdot$ RNA-seq $\cdot$ Tomato

\section{Introduction}

The translationally controlled tumor protein (TCTP), originally described as a translationally regulated protein in tumor cells, is a conserved protein found in almost all eukaryotes (Bommer and Thiele 2004). In plants, TCTP has been reported as a multifunctional protein implicated in an amazing number of cellular events, including a prominent role in cell growth and proliferation (reviewed in Gutiérrez-Galeano et al. 2014). As their mammalian counterpart, plant TCTPs are frequently reported as microtubule- and $\mathrm{Ca}^{2+}$-binding proteins (Kim et al. 2012; Hoepflinger et al. 2013; Li et al. 2013). Compelling evidences also indicate that plant TCTPs are regulated in response to a wide array of stimuli including low temperature ( $\mathrm{Li}$ et al. 2013), salt (Li et al. 2013; Santa Brígida et al. 2014), wounding (Li et al. 2013), drought 
(Ghabooli et al. 2013; Li et al. 2013), induced programmed cell death (Chen et al. 2014), mercury (Wang et al. 2015), aluminum (Ermolayev et al. 2003), hydrogen peroxide (Li et al. 2013; Wang et al. 2015) and infection by different pathogens (Alfenas-Zerbini et al. 2009; Li et al. 2013). TCTP expression is also influenced by different phytohormones, i.e., methyl jasmonate ( $\mathrm{Li}$ et al. 2013), abscisic acid (ABA) (Kim et al. 2012; Wang et al. 2015) and ethylene (Tao et al. 2015).

Two TCTP coding genes (AtTCTP1; At3g16640 and AtTCTP2; At3g05540) have been identified in the model plant Arabidopsis thaliana. Landmark studies have demonstrated the involvement of AtTCTP1 in the regulation of mitotic growth through the control of cellcycle duration (Berkowitz et al. 2008; Brioudes et al. 2010). Interestingly, the attctpl knockout mutant presented a male gametophytic phenotype with impaired pollen tube growth, while AtTCTP 1 silenced lines showed impaired root hair development and slow vegetative growth due to reduced cell size (Berkowitz et al. 2008). Surprisingly, no phenotypic alterations were observed in Arabidopsis lines overexpressing AtTCTP1 (Berkowitz et al. 2008). More recently, a putative non-cell autonomous role for AtTCTP2 was proposed based on the ability of its mRNA and protein to move long distances (Toscano-Morales et al. 2014). Moreover, a link between AtTCTP2 activity and developmental reprogramming was envisaged (Toscano-Morales et al. 2014, 2015).

Further studies revealed distinctive effects of AtTCTP1 overexpression in model plants. Transgenic overexpression of AtTCTP1 in tobacco, for example, resulted in decreased cell death, thus supporting a cytoprotective role for this protein (Hoepflinger et al. 2013). Moreover, a positive impact of AtTCTP1 overexpression in drought stress tolerance was reported in Arabidopsis (Kim et al. 2012). This exacerbated tolerance was correlated with increased ratios of ABA- and $\mathrm{Ca}^{2+}$-triggered stomatal closure and faster stomatal closing in response to ABA (Kim et al. 2012). Overall, these and other data provide direct evidence for a role of TCTP in plant stress tolerance and signaling (reviewed in GutiérrezGaleano et al. 2014).

In this regard, recent findings by Tao and colleagues (2015) have implicated a tobacco TCTP (NtTCTP) in ethylene signaling through the interaction with a previously identified type II ethylene receptor (NTHK1) involved in plant growth and stress responses (Cao et al. 2006, 2007). NtTCTP was shown to be required for the stability and proper functioning of NTHK1. Interestingly, NtTCTP-overexpressing plants showed decreased ethylene responses, which in turn led to increased seedling growth as a consequence of accelerated cell proliferation (Tao et al. 2015). Based on these results, an NtTCTP-mediated feedback mechanism for control of ethylene signaling was demonstrated.
In the present study we focused on a tomato (Solanum lycopersicum L.) TCTP coding gene (SITCTP; Gene ID 543941 ) previously reported to be induced by virus infection (Alfenas-Zerbini et al. 2009). Interestingly, effective knockdown of SITCTP in tomato resulted in reduced plant stature, abnormal fruit morphology and decreased seed size (Bruckner et al. 2016). Here, the functional relevance of SITCTP in plants was assessed by its overexpression in transgenic tobacco plants and evaluation of the resultant phenotypic alterations. Subsequently, the global gene expression changes associated with SITCTP overexpression in tobacco were examined using RNA-sequencing. In this regard, the induced transcriptomic responses revealed an important impact of SITCTP overexpression in photosynthesis and hormone responses.

\section{Materials and methods}

\section{Plant materials and growth conditions}

Tomato (S. lycopersicum cv. Santa Clara) was used for SITCTP cDNA amplification, while tobacco (Nicotiana tabacum cv. SR1) was employed for transgenic plant generation. Seeds were surface sterilized and germinated in Petri dishes containing solid MS medium (Murashige and Skoog 1962) or in pots containing soil and vermiculite (2:1). Plants were kept in a growth chamber at $22^{\circ} \mathrm{C}$ with a $16 \mathrm{~h} / 8 \mathrm{~h}$ light/dark photoperiod or in a greenhouse with controlled conditions.

\section{Total RNA extraction and reverse transcription (RT)}

Total RNA from tobacco and tomato was extracted using Trizol reagent according to the manufacturer's instructions (Invitrogen). For this, $100 \mathrm{mg}$ of macerated organ/tissue was used. The integrity of the extracted RNA was checked by electrophoresis on denaturing agarose gels and samples were stored at $-80^{\circ} \mathrm{C}$ until use. For cDNA synthesis, total RNA $(2 \mu \mathrm{g})$ was treated with RNase-free DNase I (Promega) and submitted to RT employing $2.5 \mathrm{mM}$ of oligo (dT17-VN) and the High Capacity cDNA Reverse Transcription kit (Applied Biosystems) according to the manufacturer's instructions.

\section{Amplification and cloning of the SITCTP coding region}

The SITCTP coding region (AY642284; from the ATG start codon to the stop codon) was amplified by standard PCR using cDNA prepared from tomato leaves and primers flanking the predicted SITCTP open reading frame (ORF) (TCTP-F 5'-CGGCCATGGTGTTGGTTTATCAGG-3' and TCTP-R 5'-GCCGAGCTCCTACTTGATCTC-3'). 
Primers were designed based on the nucleotide sequence of the SITCTP gene (Solyc01g099780) available at the Solanaceae Genomic Network database (http://solgenomics. net/) and supplemented with restriction sites for $\mathrm{NcoI}$ and SacI (underlined). The amplification product was digested by $N c o I$ and $S a c I$, gel purified and inserted into an equally digested intermediate cloning vector (pBI2X35S). In this plasmid, the SITCTP coding region was placed under the control of an enhanced Cauliflower mosaic virus (CaMV) $35 \mathrm{~S}$ promoter $(2 \times 35 \mathrm{~S})$ and a nopaline synthase transcriptional terminator. Ligation products were subsequently transformed into Escherichia coli competent cells. Subsequently, selected recombinant clones were sequenced using the BigDye ${ }^{\mathrm{TM}}$ Terminator v3.1 Cycle Sequencing kit (Applied Biosystems) on an ABI 3100 automated sequencer (Applied Biosystems).

\section{Generation of transgenic tobacco plants and molecular characterization}

For overexpression in transgenic tobacco plants, the expression cassette containing the full length SITCTP cDNA was transferred from the pBI2X35S derivative to the binary vector pBI121 (Clontech) using the HindIII and EcoRI sites. After sequencing, recombinant vector was introduced into Agrobacterium tumefaciens LBA4404, which was then used to transform tobacco leaf discs essentially as described (Horsch et al. 1985). Putative transformants were selected in agar plates containing MS medium supplemented with kanamycin $\left(100 \mu \mathrm{g} \mathrm{ml}^{-1}\right)$. T-DNA integration was checked by PCR employing genomic DNA extracted from leaves and primers complementary to the $35 \mathrm{~S}$ promoter (5'-ACAATCCCACTATCCTTC-3') and to the SITCTP coding region (5'-CGGCCATGGCTATGT TGGTTTATC-3'), respectively. PCR-positive plants were subsequently selected for total RNA and protein extraction to assess transgene expression.

The relative expression of SITCTP in the leaves of randomly selected transgenic tobacco plants representing independent transformation events was determined by RT-qPCR as described below. For this, a SITCTP-specific primer pair (called qTCTP) was employed (5'-AGAAGT CGAGAATGGGGTGC-3' and 5'-TGAACAACCCACTTC CCTTGA-3'). Western blot analysis was performed essentially as described (Brandalise et al. 2003) using total leaf protein extracts, a polyclonal antibody generated against bacterially expressed recombinant SITCTP (1:1000; Bruckner et al. 2016) and a peroxidase-conjugated anti-rabbit $\mathrm{IgG}$ as secondary antibody (1:2000 dilution). Plants corresponding to three transformation events showing the highest levels of transgene expression (namely TCTP2, TCTP7 and TCTP8) were further selected for use in subsequent assays. Only self-pollinated $\mathrm{T} 2$ progenies were employed.

\section{Abiotic stress treatments}

To test the behavior of the SITCTP-overexpressing (OE) plants under osmotic stress, seeds from WT and the investigated transgenic lines (TCTP2, TCTP7 and TCTP8) were surfaced sterilized and sown in Petri dishes (10 per dish) containing MS medium. Five-day-old seedlings were then transferred onto fresh MS dishes with or without mannitol supplementation $(0,100,150$ and $200 \mathrm{mM})$. The dishes (3 per treatment) were kept in vertical position in a growth chamber, and after 10 days, primary root length measurements were performed. The same procedure was used to evaluate the expression of stress-related genes in transgenic and WT seedlings. In this case, however, 10-day-old seedlings from each genotype were placed simultaneously in a single Petri dish containing half-strength MS medium supplemented with $150 \mathrm{mM} \mathrm{NaCl}$ and collected for total RNA extraction after 12 and $24 \mathrm{~h}$ of stress imposition. Unstressed seedlings were maintained in half MS medium and were collected at the same time points. To reduce plant-to-plant variation, samples from each genotype were pooled before RNA extraction.

To further evaluate the performance of the transgenic SITCTP-OE lines under osmotic and salt stress, respectively, 35-day-old plants (WT and transgenic; $n=10$ ) grown in pots were daily irrigated with $30 \mathrm{ml}$ of either $150 \mathrm{mM}$ mannitol or $175 \mathrm{mM} \mathrm{NaCl}$ for 10 days, and then allowed to recover with pure water irrigation for 3 days (Begcy et al. 2011). In the control treatment, plants were irrigated with water throughout the experiment. The fresh weight of the treated plants was immediately measured after recovery and total dry weight was recorded after drying plants in an oven at $70^{\circ} \mathrm{C}$ to a constant weight. The relative dry weight was determined as the ratio of each treated plant to its corresponding control and expressed in percentage.

\section{RNA-Seq analysis}

RNA-Seq analysis was performed using two independent SITCTP-OE lines (namely TCTP7 and TCTP8) and the WT untransformed control essentially as described previously (Laitz et al. 2015). For that, intermediary leaves were collected from five individual plants (35-day-old) per investigated genotype and then pooled to obtain representative samples. Total RNA from these independent pools was extracted using Trizol reagent (Invitrogen) as previously described (Laitz et al. 2015). RNA samples were quantified spectrophotometrically using NanoDrop (Thermo Scientific) and RNA integrity was evaluated using the RNA 6000 Nano LabChip Kit and a Bioanalyzer 2100 (Agilent Technologies). Only RNA samples with integrity number $\geq 7$ were used for library construction. The sequencing libraries were prepared using the TruSeq RNA Sample Preparation 
Kit v2 (Illumina) following manufacturer's instructions. Sequencing was performed on the Illumina MiSeq platform using a paired-end 300 base pair (bp) run. The complete dataset of RNA-Seq reads was deposited in the Sequence Read Archive (SRA) database under accession numbers SRR3099652, SRR3099654, SRR3099655, SRR3100097, SRR3100098 and SRR3100099.

\section{De novo transcriptome assembly, and mapping and differential gene expression analysis}

The transcriptome assembly was performed de novo using Trinity (Grabherr et al. 2011) allowing only contigs greater than $200 \mathrm{bp}$. The raw reads were mapped into the Trinity assembly using RSEM (Li and Dewey 2011). Differential expression analysis was performed using edgeR software (Robinson et al. 2010) with the count data from RSEM and a standard dispersion value of 0.15 .

\section{Functional annotation}

The functional annotation was performed in three steps: (1) determination of the protein-coding potential of the transcripts, (2) generation of the deduced amino acid sequences of the protein-coding transcripts and (3) assignment of Gene Ontology (GO) terms to these protein sequences. The protein-coding potential of all transcripts was determined using the support vector machine-based classifier Coding Potential Calculator (CPC; Kong et al. 2007). CPC also generated the translated sequences of the protein-coding transcripts. For the assignment of GO terms to these protein sequences, the large scale function prediction tool Argot2 (Fontana et al. 2009; Falda et al. 2012) was used with default parameters (TS score $\geq 200.0$ ). Argot 2 was selected since it was considered the top method among other existing function prediction tools as reported in a large-scale evaluation of computational protein function prediction (Radivojac et al. 2013).

\section{GO-based functional enrichment analysis}

The enrichment analysis was carried out for the proteins encoded by the differentially expressed protein-coding transcripts. For each type of GO categories, we compared the frequencies of GO terms mapped to the proteins encoded by the differentially expressed protein-coding transcripts (test set) with those of the entire set of proteins encoded by the protein-coding expressed transcripts (reference set). The Biological Networks Gene Ontology tool (BiNGO) (Maere et al. 2005) was used to determine which GO terms were significantly over represented in a set of proteins. The reference set, the ontology and organism/annotation files were all prepared in a customized way. We considered as enriched, the GO terms with frequencies 10-fold higher in the test set compared to the reference set.

\section{qPCR assays}

Quantitative PCR (qPCR) was carried out to evaluate the transcript accumulation of SITCTP (using the qTCTP primer pair) and selected stress-responsive genes (NtLEA14, NtRD29A and NtERD1; primers according to Babitha et al. 2015) in the generated transgenic tobacco lines, and also to check the accuracy of the generated RNASeq data employing a set of 17 selected genes (for details see Table S1). In the last case, the same RNA samples used for RNA-Seq library construction were employed. A gene encoding a tobacco elongation factor (NtEF; 5'-TCATTA GGTCCAGCGTTCTTAGC-3' and 5'-CCCACCCTAAGG AATCTGCAT-3') was used as endogenous control in all reactions.

The qPCR assays were performed using a Step One Plus Real-Time PCR System (Applied Biosystems) under the following parameters: $95^{\circ} \mathrm{C}$ for $10 \mathrm{~min}, 40$ cycles at $94^{\circ} \mathrm{C}$ for $15 \mathrm{~s}$ and $60^{\circ} \mathrm{C}$ for $60 \mathrm{~s}$. Amplicon specificity was checked using the dissociation curve at the end of each run. Each qPCR $(12 \mu \mathrm{l})$ consisted of $6 \mu \mathrm{l}$ of Maxima SYBR Green/ROX qPCR Master Mix (Thermo Scientific), $1 \mu \mathrm{l}$ of cDNA (1/10 dilution) and $0.4 \mu \mathrm{M}$ of forward and reserve primers, respectively. All reactions were carried out in triplicate. Relative expression data were analyzed using the DataAssist software v.3.01 (Applied Biosystems). Additionally, the correlation between the RNA-Seq and RT-qPCR expression values obtained for the selected genes was evaluated by calculating the non-parametric Spearman's correlation coefficient.

\section{Plant gas exchange parameters}

Gas exchange parameters were measured on the second youngest leaf from plants $(n=6)$ of the TCTP7 and TCTP8 OE lines and WT control, respectively. For this, an infrared gas analyzer in an open system (LCI-portable, ADC system, England) was used. Measurements were performed between 9 and 10 o'clock. Net photosynthetic rate $(A)$, intercellular $\mathrm{CO}_{2}$ concentration $\left(C_{\mathrm{i}}\right)$ and stomatal conductance $\left(g_{\mathrm{s}}\right)$ were all determined at an ambient $\mathrm{CO}_{2}$ concentration of $398 \pm 6 \mu \mathrm{mol} \mathrm{mol}{ }^{-1}$, temperature of $28 \pm 2{ }^{\circ} \mathrm{C}$, and $28 \%$ relative humidity.

\section{Results}

As a first step towards functional investigation of SITCTP, we employed Agrobacterium-mediated transformation to generate tobacco plants that overexpress 
this protein. Several independent kanamycin-resistant transformants were obtained, and the insertion of the expression cassette in eight of them was confirmed by PCR using genomic DNA extracted from leaves (not shown). Subsequent RT-qPCR analysis of the SITCTP transcripts in three randomly selected transformed lines (called TCTP2, TCTP7 and TCTP8) revealed variable levels of transgene expression (Fig. 1a). In this regard, the highest levels of transgene expression were observed in the TCTP7 and TCTP8 lines while the lowest level was observed in the TCTP2 line. A cross-amplification of the endogenous NtTCTP transcripts was observed in the WT untransformed control (relative expression set to one; Fig. 1a). The SITCTP protein content in these independent OE lines, as determined by Western blot analysis of total protein leaf extracts, was almost proportional to the level of the SITCTP transcripts (Fig. 1b). Intriguingly, the endogenous NtTCTP protein was not detected in protein extracts from WT control leaves (Fig. 1b), which might be linked to its low concentration.

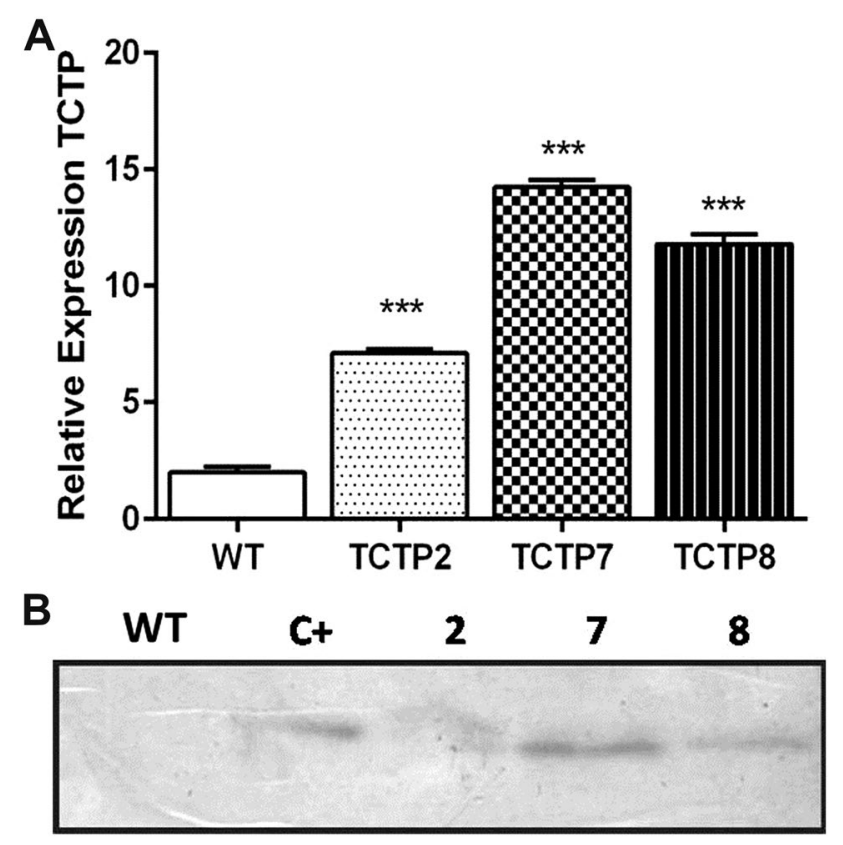

Fig. 1 Molecular characterization of the SITCTP-OE tobacco lines. a RT-qPCR analysis of transgene expression in three selected transgenic lines (TCTP2, TCTP7 and TCTP8). Values are presented as transcript abundance relative to the untransformed control (WT; set to one) and asterisks denote significant differences between WT and OE lines $(p<0.001)$. b SITCTP accumulation in the corresponding transgenic lines as determined by western blot. WT untransformed control plant, $C^{+}$bacterially expressed recombinant SITCTP
Tobacco SITCTP-overexpressing lines show increased growth

Given that most TCTPs studied so far have been implicated in the regulation of plant growth, we decided to check the growth phenotype of the aforementioned SITCTP-OE lines. In fact, as evidenced by the primary root lengths of seedlings (Fig. 2a, b) and the fresh and dry weights of adult plants (Fig. 3a, b) grown under normal conditions, the three OE lines under analysis exhibited increased growth and biomass production compared to the WT control. In line with these quantifications, an improved growth of the aerial parts of the transgenic plants relative to the WT was clearly visible (Fig. 3c, panel 1). Likewise, lateral root formation was enhanced in the SITCTP-OE seedlings compared to the WT (supplementary Fig. S1), suggesting an improved root growth.

Overall, a positive correlation between SITCTP overexpression and plant growth could be observed. In this regard, pair-wise comparisons between the transgenic lines revealed that, under control conditions, the lines

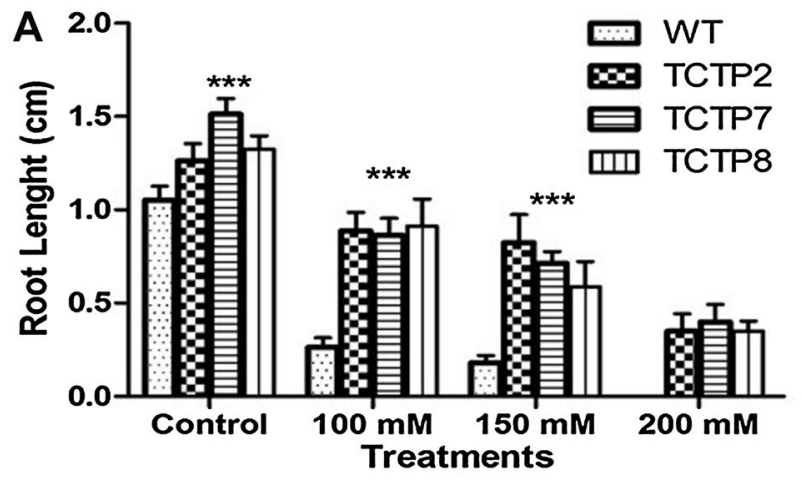

B

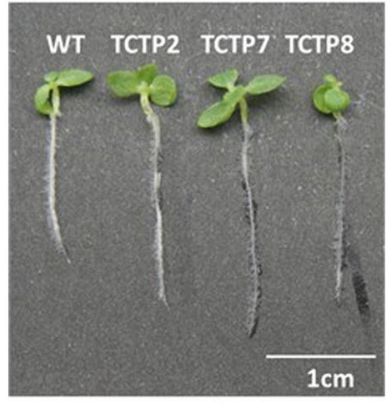

C

Control

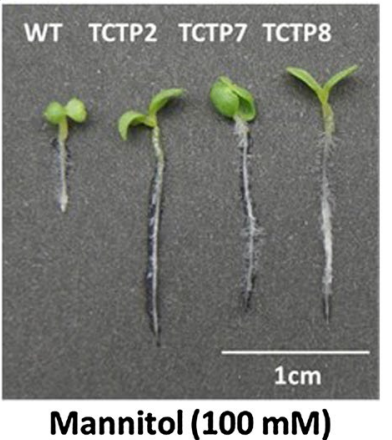

Fig. 2 Root phenotypes of the SITCTP-OE lines. a Primary root length of WT and OE seedlings grown under control condition or upon exposure to osmotic stress (100, 150 and $200 \mathrm{mM}$ mannitol). Data are the means from three independent experiments using ten seedlings \pm SE. Asterisks indicate significant differences between WT and OE lines as defined by Kruskal-Wallis test $(* p<0.05$ and $* * * p<0.001)$. Photographs of representative seedlings (15-day-old) from each genotype grown under control (b) and mannitol-stressed $(100 \mathrm{mM})(\mathbf{c})$ conditions 

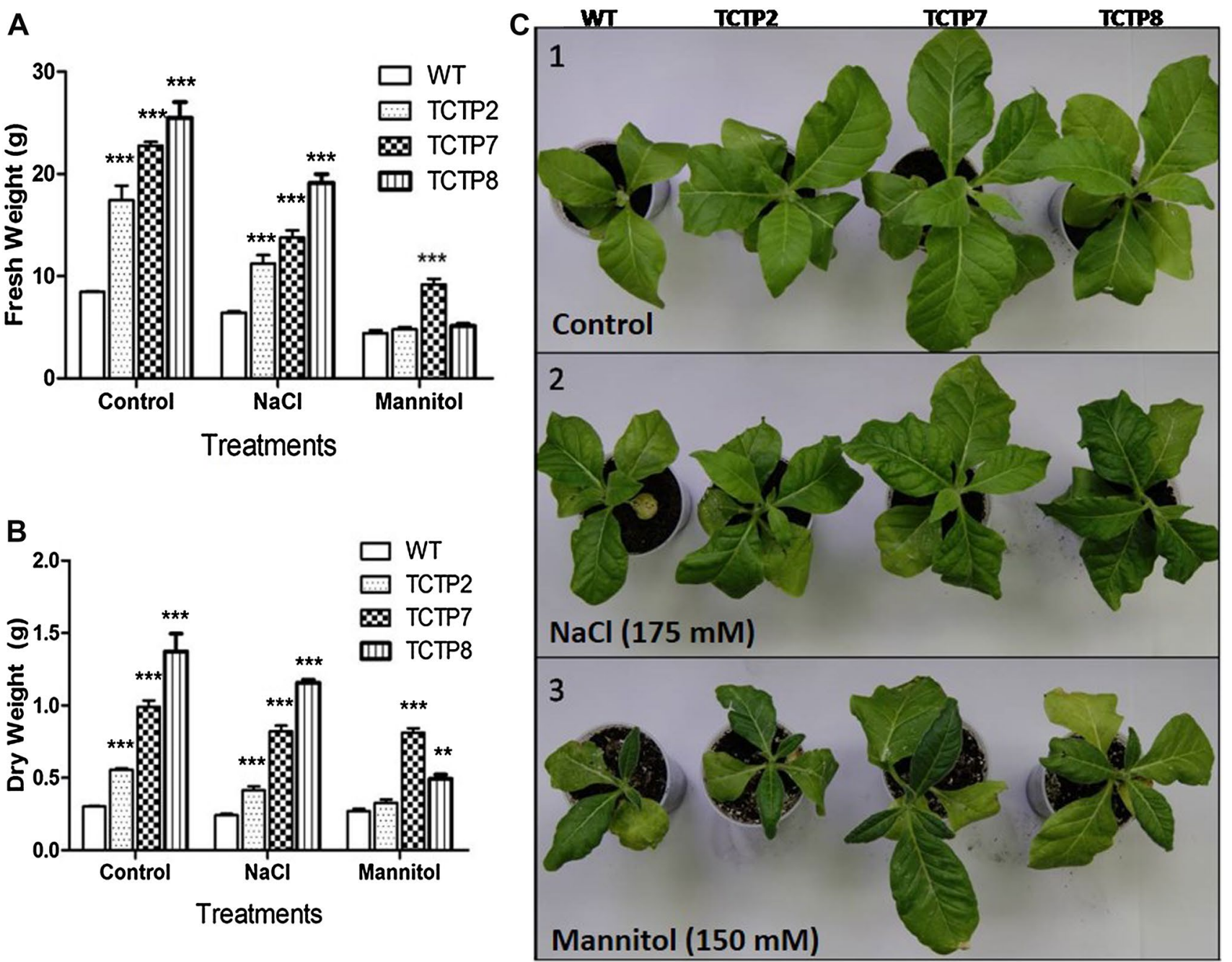

Fig. 3 Biomass accumulation of WT and SITCTP-OE plants under stress. Fresh (a) and dry (b) weights of the WT and OE plants (35-day-old) submitted to control, salt and osmotic stress conditions, respectively. Data are the means from three independent experiments using ten plants \pm SE. Asterisks indicate significant differ- ences between WT and OE lines as defined by Kruskal-Wallis test $(* * p<0.01$ and $* * * p<0.001)$. c Photographs of representative plants from the tested genotypes taken at the end of each treatment, i.e. (1) control condition, (2) salt stress $(175 \mathrm{mM} \mathrm{NaCl})$ and (3) osmotic stress (150 mM mannitol) with high transgene expression levels (namely TCTP7 and TCTP8) presented significantly higher fresh and dry weights $(p<0.05)$ when compared to line TCTP2, which has the lowest transgene expression level (Fig. 3a, b). On the other hand, the detected differences in both fresh and dry weights between lines TCTP7 and TCTP8 were not statistically significant (Fig. 3a, b). Interestingly, an opposite behavior was detected in transgenic tomato lines with strong knockdown of SITCTP expression (about $94-97 \%$ of the WT level), which exhibited stunted growth and delayed development compared to WT plants (Bruckner et al. 2016).

\section{SITCTP-OE lines show better performance under abiotic stress}

To determine the impact of SITCTP overexpression in plant performance under abiotic stress, WT and transgenic seedlings were first subjected to osmotic stress using mannitol, and root elongation was used as an indicator of stress sensitivity. As a result, the observed differences in primary root length between the tested OE lines and the WT control grown under non-stressed conditions were even more pronounced when seedlings were submitted to osmotic stress (Fig. 2a, c). According to the quantifications shown in 
Fig. 2a, the roots of the transgenic lines were less sensitive to the osmotic stress than those of the WT control. Moreover, in the presence of $200 \mathrm{mM}$ mannitol, a severe inhibition of root growth was observed in the WT control relative to the OE lines subjected to the same treatment.

Transgenic plant performance under stress was further evaluated using adult plants submitted to osmotic and salt stresses, respectively. As indicated by their higher fresh and dry weights under saline conditions (Fig. 3a, b), the OE plants were clearly less sensitive to salt stress than the WT plants. Relative dry weights under salt stress decreased from 14 (TCTP8) to 20\% (TCTP2 and TCTP7) in the transgenic OE lines, while a $25 \%$ reduction was detected in WT, indicating that the transgenic lines sustained a relatively better growth than WT. Moreover, as documented in Fig. 3c (panel 2), the visual symptoms of salt stress damage were less intense in the transgenic plants, indicating that these plants were not significantly compromised by the applied stress. Overall, these results indicate that plant performance under salinity was improved by the ectopic overexpression of SITCTP. Moreover, this is the first demonstration of a beneficial effect of a plant TCTP on salt tolerance at the whole plant level.

On the other hand, compared with WT plants, only the OE line with highest transgene expression level (TCTP7) displayed better growth performance under mannitolinduced osmotic stress (Fig. 3a, b). By contrast, as indicated by their biomass accumulation, the moderate overexpressing TCTP8 line displayed an intermediate behavior between WT and TCTP7, while the lower overexpressing TCTP2 line was as sensitive to osmotic stress as the WT control (Fig. 3a, b). The observed reductions in relative dry weights ranged from 37 (WT) to 64\% (TCTP8). As aforementioned, only TCTP7 retained high biomass under osmotic stress with a $20 \%$ reduction in relative dry weight. Therefore, a negative correlation between SITCTP expression levels and sensitivity to osmotic stress was observed.

To determine whether the better performance of the SITCTP-OE lines under abiotic stress was associated with an altered expression of stress-responsive genes, we decide to evaluate the expression of three known stressmarker genes (NtLEA14, NtRD29A and NtERDI) in WT and $\mathrm{OE}$ seedlings grown under control (unstressed) and salt-stressed conditions (Fig. 4). To give further confidence to our data, results were considered of significance only if a similar expression trend over WT was observed in all tested OE lines. In this context, NtRD29A was significantly down-regulated (about threefold) in all SITCTP-OE lines under control conditions (12 h) (Fig. 4a). On the other hand, compared to WT, NtLEA14 transcript levels were increased by six or more folds in the three OE lines after $24 \mathrm{~h}$ of salt treatment (Fig. 4b). Concerning NtERD1, this gene was down-regulated in control unstressed conditions
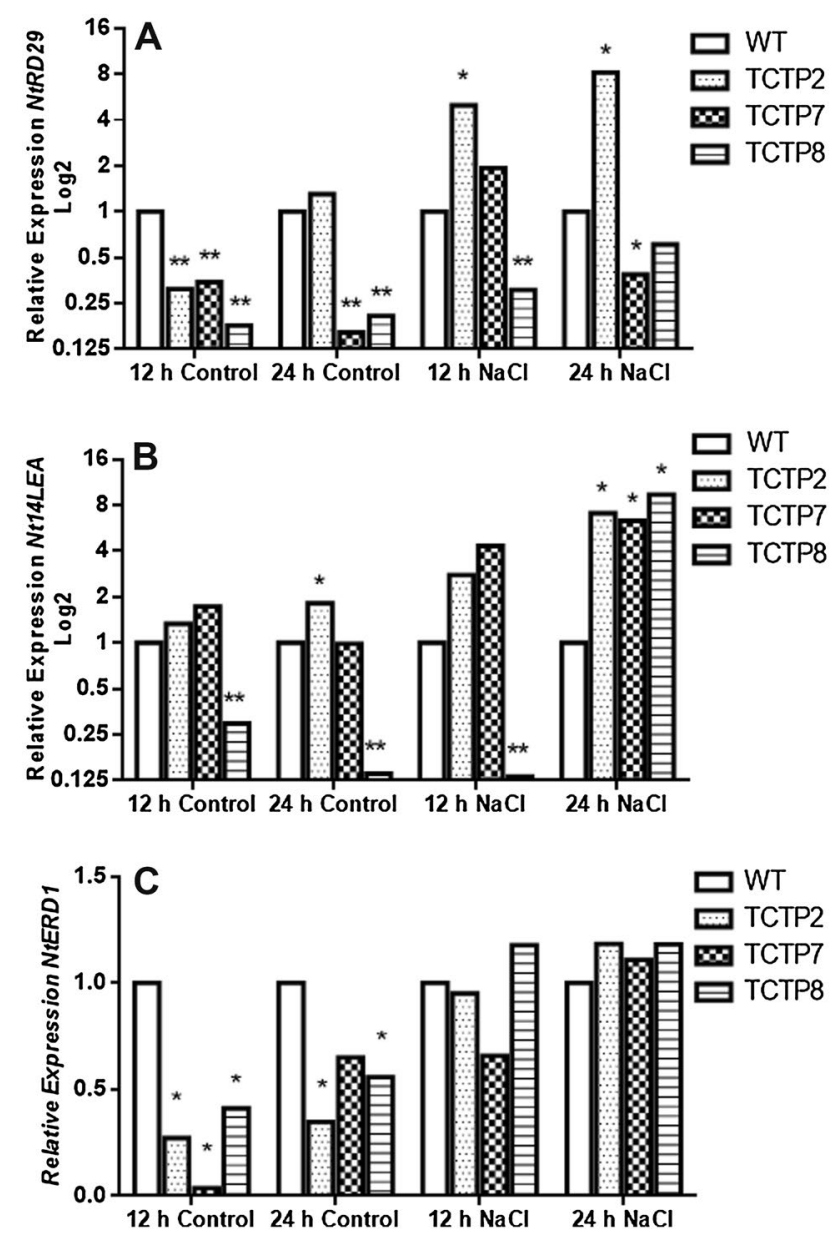

Fig. 4 Fold change in expression of selected stress-related genes in the SITCTP-OE lines (TCTP2, TCTP7 and TCTP8) over WT plants. a NtRD29A ( $\log _{2}$ transformed); b NtLEA14 ( $\log _{2}$ transformed) and c NtERD1. Transgenic and WT seedlings $(n=5)$ were treated with $150 \mathrm{mM} \mathrm{NaCl}$ and sampled at 12 and $24 \mathrm{~h}$ of stress imposition. Control (unstressed) seedlings were sampled at the same time points. Values of WT expression were set to 1. Asterisks indicate a significant difference from the WT at the corresponding condition (Student's $t$ test; $* p<0.05$ and $* * p<0.01$ )

(12 h) in all transgenic lines and remained unchanged over WT under salt treatment (Fig. 4c). Overall, these findings indicate that the positive effects of SITCTP overexpression on plant performance under salt stress is not paralleled with a consistent up-regulation of the investigated stress-related genes. These results also suggest that other mechanisms, which probably require the concerted stimulation of cellular and physiological processes by SITCTP, are involved.

\section{RNA-Seq analysis suggests altered photosynthesis in the $\mathrm{OE}$ lines}

To gain a better understanding of the molecular mechanisms associated with the observed effects of SITCTP in plant growth and stress responses, a transcriptome analysis 
of the independent $\mathrm{OE}$ lines with major transgene expression (TCTP7 and TCTP8) was conducted using RNA-Seq. For that, pools of leaves collected from five independent WT and transgenic plants, respectively, were used. Approximately 14 million raw paired-end sequencing reads, $151 \mathrm{bp}$ on average, were generated and assembled de novo. After quality check and data cleaning, 7,612,562 high quality reads were obtained with $99.6 \%$ Q20 bases (base quality more than 20). A total of 122,389 contigs were assembled with an average length of $656,64 \mathrm{bp}$ (Table 1). Among the total assembled contigs, 71,668 (58.6\%) and 45,666 $(37.3 \%)$ presented a positive match in searches against the NCBI and Swiss-Prot databases ( $E$ value: $1 E-5)$, respectively. On the other hand, 55,349 (45\%) were identified as protein-coding transcripts using CPC (Kong et al. 2007), and among them, 53,590 presented a positive BLASTP match in searches against the UniProtKB database. Additionally, using Argot2, 36,670, 33,278 and 38,710 were assigned with at least one GO term in either biological process, cellular component or molecular function categories, respectively.

The differentially expressed genes (DEGs) were identified by comparing the RNA-Seq data obtained for the nontransformed WT plants and the data of each transgenic OE line. The criteria used to select these DEGs were FDR values $\leq 0.01$ and RPKM values $\geq 1$, respectively. As a result, 215 transcripts were identified as differentially regulated between WT and TCTP7, while 180 transcripts were identified between WT and TCTP8. Among them, 87 were found to be common to both $\mathrm{OE}$ lines. To instill further confidence in our RNA-Seq data, and considering the high correlation observed between the generated dataset of the two OE lines (Supplementary Fig. S2), only these common DEGs were used in the subsequent analysis. Out of these, 59 were considered up-regulated $(\geq 0.3 \log$ fold change) while 28 were down-regulated ( $\leq 0.3$ log fold change) (Supplementary Tables S2 and S3).

A categorization of the common up- and down-regulated DEGs was then carried out employing established GO categories. It should be mentioned here that in many cases, different GO terms were assigned to the same transcript. Among the up-regulated DEGs allocated in the biological

Table 1 Summary of the generated RNA-Seq data

\begin{tabular}{lr}
\hline Transcripts & 122,389 \\
Mean transcript length (bp) & 656,64 \\
Bigger transcript (bp) & 10,663 \\
Smaller transcript (bp) & 201 \\
Transcripts ( $\geq 500 \mathrm{bp})$ & 50,222 \\
Annotated components (Uniref90) & 71,669 \\
Annotated components (Swiss-Prot) & 45,666 \\
\hline
\end{tabular}

process category, the majority is involved in biosynthetic processes (21 transcripts) and photosynthesis (16 transcripts) (Supplementary Fig. S3). Also within this category, genes associated with transport (14 transcripts), response to stress (13 transcripts) and response to abiotic stimulus (14 transcripts) were observed (Supplementary Fig. S3). The most representative terms in the molecular function category were oxidoreductase activity (10 transcripts), organic cyclic compound binding (10 transcripts) and heterocyclic compound binding (10 transcripts). Another term that stands out in this category is hydrolase activity ( 9 transcripts) (Supplementary Fig. S3). The up-regulated DEGs allocated in the cellular component category were found in the chloroplast thylakoid membrane (15 transcripts), chloroplast stroma (12 transcripts) and chloroplast envelope (10 transcripts) (Supplementary Fig. S3).

In the set of down-regulated DEGs, the most represented GO terms in the biological process category were oxidation-reduction process (10 transcripts) and response to stress (8 transcripts) (Supplementary Fig. S4), while oxidoreductase activity (11 transcripts) was the most represented term in the molecular function category. This category was also overrepresented by several binding terms including metal ion binding (11 transcripts), anion binding (6 transcripts) and nucleotide binding (5 transcripts) (Supplementary Fig. S4). The down-regulated genes were almost equally found in the cytoplasm, membrane and intracellular membrane-bounded organelle (Supplementary Fig. S4).

\section{Functional enrichment confirms overrepresentation of photosynthesis-associated terms}

An enrichment analysis was performed to identify the significantly overrepresented GO terms associated with the up-regulated and down-regulated gene datasets. Within the up-regulated gene dataset, the most significantly enriched terms for biological processes were photosynthesis and lipid metabolic process (Table 2). Consonant with the detected enrichment of photosynthesis-related terms, the majority of the GO terms significantly enriched in the cellular component category was associated with the chloroplast (chloroplast envelope; chloroplast thylakoid; chloroplast thylakoid membrane; chloroplast thylakoid lumen) or linked to the photosystems (PS) I and II or to the PSI reaction center (Table 2). Additional functional sites were cell wall and extracellular region. Under the molecular function category, transporter activity followed by oxidoreductase activity acting in paired donors, protochlorophyllide reductase activity and hydrolase activity were markedly enriched (Table 2).

On the other hand, a closer look to the down-regulated gene dataset revealed enrichment in GO terms associated 
Table 2 Significantly enriched GO terms for the up-regulated DEGs

\begin{tabular}{|c|c|c|c|c|c|}
\hline GO-ID & Term & $p$ value & Corrected $p$ value & $\mathrm{x} / \mathrm{n}(\%)$ & $\mathrm{X} / \mathrm{N}(\%)$ \\
\hline $9579^{\mathrm{a}}$ & Thylakoid & $5.8433 E-7$ & $1.8699 E-5$ & (6/43) $13.9 \%$ & $(236 / 33,278) 0.7 \%$ \\
\hline $9534^{\mathrm{a}}$ & Chloroplast thylakoid & $4.7658 E-6$ & $6.1733 E-5$ & $(5 / 43) 11.6 \%$ & $(191 / 33,278) 0.5 \%$ \\
\hline $9535^{\mathrm{a}}$ & Chloroplast thylakoid membrane & $5.7874 E-6$ & $6.1733 E-5$ & (6/43) $13.9 \%$ & $(351 / 33,278) 1.0 \%$ \\
\hline $9538^{\mathrm{a}}$ & Photosystem I Reaction Center & $1.6942 E-4$ & $1.3554 E-3$ & (2/43) $4.6 \%$ & $(15 / 33,278) 0.0 \%$ \\
\hline $9523^{\mathrm{a}}$ & Photosystem II & $5.2036 E-4$ & $3.3303 E-3$ & $(3 / 43) 6.9 \%$ & $(121 / 33,278) 0.3 \%$ \\
\hline $9941^{\mathrm{a}}$ & Chloroplast envelope & $1.1596 E-3$ & $6.1847 E-3$ & $(5 / 43) 11.6 \%$ & $(617 / 33,278) 1.8 \%$ \\
\hline $9543^{\mathrm{a}}$ & Chloroplast thylakoid lumen & $1.6281 E-3$ & $7.4427 E-3$ & (2/43) $4.6 \%$ & $(46 / 33,278) 0.1 \%$ \\
\hline $9522^{\mathrm{a}}$ & Photosystem I & $2.4027 E-3$ & $9.6110 E-3$ & (2/43) $4.6 \%$ & $(56 / 33,278) 0.1 \%$ \\
\hline $5618^{\mathrm{a}}$ & Cell wall & $3.0423 E-3$ & $1.0817 E-2$ & (4/43) $9.3 \%$ & $(466 / 33,278) 1.4 \%$ \\
\hline $9507^{\mathrm{a}}$ & Chloroplast & $4.1642 E-3$ & $1.3326 E-2$ & (11/43) $25.5 \%$ & $(3511 / 33,278) 10.5 \%$ \\
\hline $9536^{\mathrm{a}}$ & Plastid & $1.6355 E-2$ & $4.5471 E-2$ & (7/43) $16.2 \%$ & $(2079 / 33,278) 6.2 \%$ \\
\hline $16020^{\mathrm{a}}$ & Membrane & $1.7931 E-2$ & $4.5471 E-2$ & (14/43) $32.5 \%$ & $(6097 / 33,278) 18.3 \%$ \\
\hline $5576^{\mathrm{a}}$ & Extracellular region & $1.8473 E-2$ & $4.5471 E-2$ & (4/43) $9.3 \%$ & $(787 / 33,278) 2.3 \%$ \\
\hline $15979^{\mathrm{b}}$ & Photosynthesis & $2.3940 E-6$ & $1.2688 E-4$ & $(5 / 48) 10,4 \%$ & $(163 / 36,670) 0.4 \%$ \\
\hline $6629^{b}$ & Lipid metabolic process & $1.7271 E-4$ & $4.5769 E-3$ & $(6 / 48) 12,5 \%$ & $(634 / 36,670) 1.7 \%$ \\
\hline $5215^{\mathrm{c}}$ & Transporter activity & $5.2593 E-7$ & $2.4719 E-5$ & $(8 / 46) 17.3 \%$ & $(605 / 38,710) 1.5 \%$ \\
\hline $16717^{\mathrm{c}}$ & Oxidoreductase activity & $9.1572 E-6$ & $2.1519 E-4$ & $(3 / 46) 6.5 \%$ & $(34 / 38,710) 0.1 \%$ \\
\hline $16630^{\mathrm{c}}$ & Protochlorophyllide reductase A & $3.2366 E-4$ & $3.4414 E-3$ & (2/46) $4.3 \%$ & $(6 / 38,710) 0.0 \%$ \\
\hline $16788^{\mathrm{c}}$ & Hydrolase activity & $3.4414 E-3$ & $3.3472 E-2$ & $(3 / 46) 6.5 \%$ & (254/38,710) $0.6 \%$ \\
\hline $46422^{\mathrm{c}}$ & Violaxanthin de-epoxidase A & $3.5608 E-3$ & $3.3472 E-2$ & (1/46) $2.1 \%$ & (3/38,710) $0.0 \%$ \\
\hline
\end{tabular}

$x / n$ number of genes in each category relative to the total used in the analysis, $X / N$ number of genes in each category relative to the total used in functional annotation

${ }^{\mathrm{a}}$ Cellular component

${ }^{\mathrm{b}}$ Biological process

${ }^{\mathrm{c}}$ Molecular function

with oxidoreductase activity, oxidation-reduction and pore complex in the molecular function, biological process and cellular component categories, respectively (Table 3). Overall, these results indicate an important effect of SITCTP overexpression on chloroplast metabolism and suggest a close relationship between TCTP activity and plant photosynthetic capacity. Another interesting feature was the down-regulation of genes encoding different isoforms of 1-aminocyclopropane-1-carboxylate (ACC) oxidase, a rate-limiting enzyme in the ethylene biosynthetic pathway. ACO4, for example, showed a significant down-regulation in both investigated OE lines (Supplementary Fig. S6). These results suggest that ethylene synthesis might be deregulated in the SITCTP OE plants, which is consistent with the reported role of TCTP as a negative regulator of ethylene responses in tobacco (Tao et al. 2015).

\section{Gene expression validation by RT-qPCR}

On the basis of the GO enrichment analysis described above, nine up-regulated and eight down-regulated genes (Supplementary Table S1) were selected for further gene
Table 3 Significantly enriched GO terms for the downregulated DEGs

\begin{tabular}{llllll}
\hline GO-ID & Description & $p$ value & Corrected $p$ value & x/n $(\%)$ & X/N (\%) \\
\hline $46930^{\mathrm{a}}$ & Pore complex & $5.41 E+00$ & $1.08 E+02$ & $(1 / 18) 5.5 \%$ & $(1 / 33,278) 0.0 \%$ \\
$55114^{\mathrm{b}}$ & Oxidation reduction process & $2.80 E-02$ & $8.12 E-01$ & $(10 / 25) 40.0 \%$ & $(2511 / 36,670) 6.8 \%$ \\
$16491^{\mathrm{c}}$ & Oxidoreductase activity & $2.25 E-02$ & $9.66 E-01$ & $(10 / 26) 38.4 \%$ & $(2468 / 38,710) 6.3 \%$ \\
\hline
\end{tabular}

$x / n$ number of genes in each category relative to the total used in the analysis, $X / N$ number of genes in each category relative to the total used in functional annotation

${ }^{\mathrm{a} C}$ Cellular component

${ }^{\mathrm{b}}$ Biological process

${ }^{\mathrm{c}}$ Molecular function 
expression validation using RT-qPCR. For that, the same RNA samples used for library construction were employed. In general, a positive correlation between the expression data derived from the RT-qPCR and RNASeq analyses was observed for each investigated OE line (Supplementary Fig. S5), thus confirming the reliability of the generated data.

Of the nine over-expressed genes chosen for validation, five $(55.5 \%)$ were significantly up-regulated in both transgenic OE lines compared to the WT (Supplementary Fig. S6). Amongst them, three are associated with photosynthetic pathways, i.e. VDE (encoding a violaxanthin de-epoxidase), PSAH (encoding a PSI reaction center subunit) and POR (encoding a protochlorophyllide reductase involved in chlorophyll biosynthesis), one encodes a tonoplast intrinsic aquaporin $(T I P 1)$ and the last one a fatty acid desaturase $(F A D 3 C)$. Among the validated down-regulated genes, four of them $(50 \%)$, namely ACO4, C7IAI, INO1 and PERX, showed significant down-regulation in both investigated OE lines, while two (25\%) (CIPK6 and NCPR) were down-regulated in at least one transgenic line (Supplementary Fig. S6). Thus, similar expression patterns of the validated genes in both or in at least one of the tested OE lines were observed.

\section{SITCTP OE lines show increased photosynthetic capacity}

Given the detected up-regulation of photosynthesisrelated genes in our transcriptomic profiling, we checked whether the SITCTP-OE lines have altered photosynthetic capacity. For this, gas exchange parameters were measured in attached leaves of the transgenic (TCTP7 and TCTP8) and WT plants grown under normal conditions. As shown in Fig. 5a, b, net photosynthesis $(A)$ and stomatal conductance $\left(g_{\mathrm{s}}\right)$ were significantly higher in the SITCTP-OE plants than in WT control plants. Unexpectedly, a difference in $g s$ values between the transgenic lines was detected. In addition, compared to the WT, $\mathrm{CO}_{2}$ concentration in the sub-stomatal chamber $\left(C_{\mathrm{i}}\right)$ was significantly reduced in both $\mathrm{OE}$ lines (Fig. $5 \mathrm{c}$ ). The $\mathrm{OE}$ lines also displayed a higher $A / C_{\mathrm{i}}$ ratio at ambient $\mathrm{CO}_{2}$ concentration (Fig. 5d) and enhanced electron transport rates compared to the WT (Fig. 5e). Taken together, these results clearly indicate that SITCTP overexpression enhanced the photosynthetic rates of the transgenic plants, which is consistent with the observed positive effect of SITCTP overexpression in plant biomass production. Surprisingly, the observed differences were not directly correlated with the level of SITCTP expression.

\section{Discussion}

TCTP has been reported as a multifunctional protein involved in a myriad of cellular processes (reviewed in Nagano-Ito and Ichikawa 2012). Despite being extensively investigated in different models, the molecular mechanisms underlying this supposed multifunctionality remain poorly understood. In plants, available data document the importance of TCTP in cell proliferation and cell reprogramming (Berkowitz et al. 2008; Brioudes et al. 2010; Toscano-Morales et al. 2014, 2015). Intriguingly, although presenting significant levels of sequence and structural conservation, the existence of functional specialization in plants has been suggested (Gutiérrez-Galeano et al. 2014). However, this issue remains largely unresolved. To gain further insights into TCTP function in planta, in this study we report on the consequences of the overexpression in tobacco of a TCTP from tomato (SITCTP).

The overexpression of SITCTP produced important phenotypic alterations in transgenic tobacco plants, including increased root growth and aerial biomass production. These effects were observed in both normal and stressed growth conditions. Intriguingly, the growth-promoting effects of overexpressed plant TCTPs are still a matter of debate. In line with our results, Tao and colleagues (2015) also observed an increase in seedling growth as well as, in the size of leaves of transgenic tobacco lines overexpressing NtTCTP. The authors concluded that this effect was directly associated with the function of NtTCTP as a negative regulator of the ethylene response, which results in acceleration of the cell cycle and cell proliferation. Overexpression of AtTCTP2 in tobacco also increased plant size, but this phenotype was only visible until 45 days post germination (Toscano-Morales et al. 2015), suggesting an accelerate growth rate phenotype rather than a direct effect on net growth. In contrast, no obvious changes in plant growth could be observed in AtTCTP1-overexpressing Arabidopsis lines (Berkowitz et al. 2008; Kim et al. 2012). These discrepancies might be attributed to the level of transgene expression in these different plant models. Of note, since TCTP is highly regulated at the translational level, its accumulation in these transgenic lines may vary significantly as already evidenced for AtTCTP1 in Arabidopsis (only 50\% increase at the protein level despite a 13-fold increase in gene expression; Berkowitz et al. 2008). Consistent with this, the severity of the phenotypic alterations associated with the silencing of SITCTP in tomato was directly correlated with the efficiency of gene knockdown (Bruckner et al. 2016). Alternatively, the additive effect of the endogenous NtTCTP on the growth of the SITCTP-OE plants could be envisaged.

Supporting the idea that plant TCTPs present overlapping functions in plant growth and stress response, in 

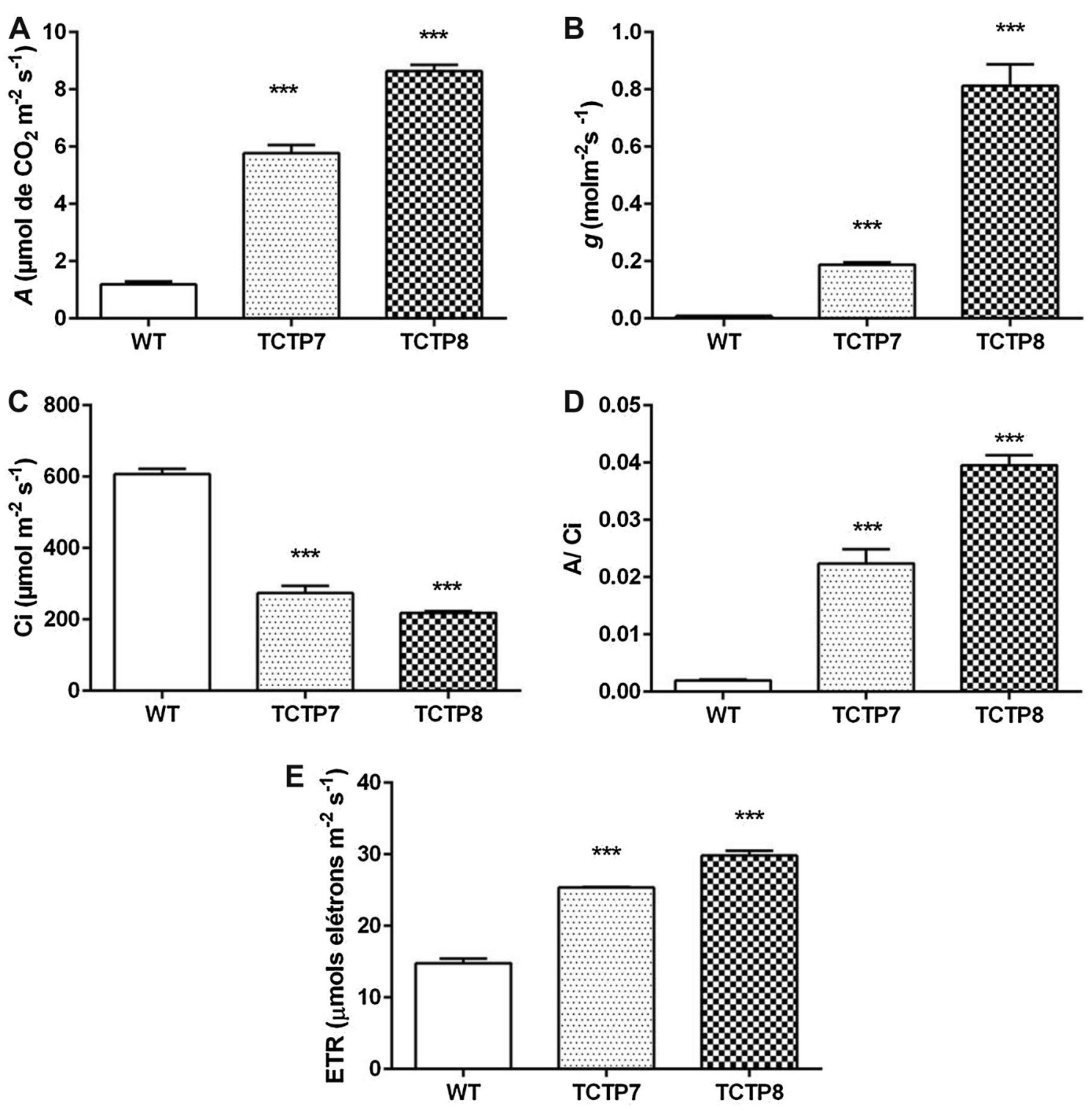

Fig. 5 Gas exchange analyses of the SITCTP-OE lines (TCTP7 and TCTP8) and WT control. a Net photosynthetic rate $(A)$; b stomatal conductance $\left(g_{\mathrm{s}}\right) ; \mathbf{c} \mathrm{CO}_{2}$ concentration in the sub-stomatal cham- ber $\left(C_{\mathrm{i}}\right) ; \mathbf{d} A / C_{\mathrm{i}}$ ratio and e electron transport rate (ETR). Data are mean $\pm \mathrm{SE}(n=15)$. Asterisks denote significant differences between WT and OE lines ( $* p$ value between 0.01 and 0.05 ; ***p $p<0.0001$ ) addition to improved biomass production, the SITCTP-OE lines also displayed better performance under abiotic stress conditions. Despite being first observed in bacterial cells (Santa Brígida et al. 2014), here we show for the first time that the overexpression of a plant TCTP is able to reduce salt-stress sensitivity at the whole plant level. Similarly, SITCTP overexpression improved plant performance under osmotic stress. Even though such response was linked to the level of transgene expression, this observation is consistent with previous data showing enhanced drought tolerance and cytoprotective effects associated with the overexpression of AtTCTP1 in Arabidopsis (Kim et al. 2012; Hoepflinger et al. 2013). Our findings are also corroborated by the reported roles of TCTP in ABA and ethylene signaling pathways (Kim et al. 2012; Tao et al. 2015), which play important roles in abiotic stress adaptation (Cao et al. 2007; Anderson et al. 2004). Therefore, regardless of the exact molecular mechanism involved, it is entirely plausible that all these TCTP-associated features accounted for the manifested better performance of the SITCTP-OE lines under stress. In this context, TCTP-mediated acceleration of plant growth would be one such mechanism that alleviates drought and salt stress effects.

To give clues to our understanding of how SITCTP affects plant growth and plant performance under stress, we analyzed the transcriptome of the transgenic SITCTP-OE 
lines and evaluated the altered gene expression profiles compared to WT plants. It should be highlighted that, to ensure confidence, only DEGs that were in common between both $\mathrm{OE}$ lines were considered. We already used such strategy in a previously published paper with similar objectives (Laitz et al. 2015). The first interesting picture that emerged from this global transcriptome profiling was the up-regulation of photosynthetic-related genes, including those involved in the photosystem reaction centers, electron transport, light reactions, and protection against photo-inhibitory damage. This was further substantiated by the significant increase in transcript accumulation of three selected photosynthetic-related genes (VDE, POR and $P S A H$ ) in the SITCTP-OE lines as determined by RTqPCR. These findings indicate that TCTP overexpression promotes a stimulatory effect on photosynthesis. Corroborating this hypothesis, we demonstrated that the SITCTPOE lines presented significantly higher rates of photosynthesis and stomatal conductance relative to WT control plants. This could be interpreted as a need to improve energy supply to sustain the detected increase in transgenic plant biomass production. On the other hand, high photosynthetic rate and stomatal conductance have been frequently associated with improved abiotic stress tolerance in different plant species (Moradi and Ismail 2007; Chaves et al. 2009).

Another well-represented group within the up-regulated dataset was composed of genes coding for different members of the aquaporin family (in special PIPs and TIPs). Aquaporins are known to contribute to the adjustment of water flux and ion homeostasis, particularly under stress (Maurel et al. 2015). Several evidences support the idea that aquaporins are also able to control water flux into guard cells, thereby regulating stomatal movement. In fact, a plasma membrane intrinsic aquaporin (PIP2.1) has been recently shown to be required for ABA-dependent stomatal regulation (Grondin et al. 2015), a function that is compatible with the reported role of TCTP in ABA-induced stomatal closure (Kim et al. 2012). Likewise, the global down-regulation of PIP expression in poplar increased the abundance of a TCTP and resulted in deregulation of stomatal control with excessive stomatal opening ( $\mathrm{Bi}$ et al. 2015).

Giving further support to the involvement of TCTP in ABA-mediated stomatal movement, we also detected a significant up-regulation of a gene encoding an aspartic protease in guard cells $(A S P G)$, which is implicated in drought tolerance through ABA signaling in guard cells (Yao et al. 2012), and a remarkable down-regulation of a gene encoding $\beta$-amylase 1 (BAM1), an enzyme that catalyzes stomatal starch degradation during guard cell opening (Valerio et al. 2011). Intriguingly, a baml knockout mutant showed decreased stomata aperture, due to impaired starch breakdown, and consequently improved drought tolerance (Prasch et al. 2015). As an additional evidence linking SITCTP to ABA-mediated responses, we observed the down-regulation of CIPK6, a calcineurin B-like (CBL)interacting protein kinase implicated in $\mathrm{ABA}$ signaling. CIPK6, when overexpressed in Arabidopsis, rendered the transgenic plants salt-tolerant and hypersensitive to ABA (Chen et al. 2012). Overall, these findings point to TCTP as a key mediator of ABA-dependent regulation of stomatal movement, a function that seems to require alterations in the abundance of a subset of aquaporins and the downregulation of BAMI (Prasch et al. 2015).

SITCTP overexpression was also accompanied by the up-regulation of genes coding for enzymes involved in fatty acid (FA) metabolism, in especial desaturases (such as Omega-3 fatty acid desaturase and Delta(8)-fatty acid desaturase) implicated in FA unsaturation. This finding is particularly striking since unsaturated FAs have been implicated in drought and defense responses (reviewed in Upchurch 2008). The increase in FA desaturation through the overexpression of two $\omega-3$ desaturases (namely FAD3 and FAD8), for example, was found to enhance osmotic stress tolerance in tobacco (Zhang et al. 2005). These data suggest that TCTP overexpression is likely associated with a higher accumulation of unsaturated FA, a hypothesis that should be further investigated. Noteworthy, polyunsaturated FAs may also serve as precursors for the synthesis of jasmonate (JA), which acts as a signaling molecule in defense responses. In this context, a gene encoding a plastid allene oxide synthase, which catalyzes the first committed step of JA biosynthesis, was found as specifically up-regulated in the SITCTP transgenic lines.

On the other hand, our transcriptome analysis revealed the down-regulation of genes encoding different isoforms of ACO as well as, a peroxisomal catalase (CAT2) involved in reactive oxygen species scavenging. Interestingly, an Arabidopsis cat 2 knockout mutant exhibited ethylene insensitivity and reduced ethylene production compared to WT plants (Bueso et al. 2007). These results suggest that ethylene synthesis might be deregulated in the SITCTP$\mathrm{OE}$ lines, what is in accordance with the reported involvement of the tobacco TCTP in ethylene signaling (Tao et al. 2015). In NtTCTP-silenced tobacco lines, an enhanced expression of genes involved in ethylene signal transduction and biosynthesis was observed (Tao et al. 2015). It should be emphasized that SITCTP shows $80 \%$ amino acid identity with NtTCTP. Also noteworthy, some of the described phenotypic alterations caused by the silencing of SITCTP in tomato, such as stunting, modified inflorescence habit and abnormal fruit morphology (Bruckner et al. 2016), resemble ethylene-associated pleiotropic effects reported in tomato (Lin et al. 2008). In addition, the fact that the SITCTP-OE lines exhibited enhanced lateral root 
formation could be also a result of altered hormone content and signaling.

Strikingly, according to our RNA-Seq data, genes associated with cell cycle regulation were not significantly affected by SITCTP overexpression. This was unexpected considering the evidenced roles of NtTCTP and AtTCTP1 in the control of cell cycle progression (Brioudes et al. 2010; Tao et al. 2015), and of AtTCTP1 in the regulation of the TOR signaling through the interaction with Rab GTPases (Berkowitz et al. 2008; Brioudes et al. 2010). One possible explanation is that SITCTP may exert such regulatory roles at the post-transcriptional or translational levels.

In conclusion, we provide evidence that SITCTP overexpression promotes important alterations in plant growth and performance under stress, which correlates with positive changes in the transcriptional profiles of genes involved in photosynthesis, fatty acid metabolism and water transport. The transcriptional modulation of genes associated with the control of stomatal movement through ABA signaling was also observed. In contrast, TCTP overexpression negatively affected the expression of genes involved mainly in ethylene biosynthesis.

Author contribution statement $\mathrm{MC}$ and IGM conceived the work and wrote the manuscript. MC, AVNL, LMA and MLCA performed the experiments. MLA and LCN performed the bioinformatics analysis and revised the manuscript. All authors read and approved the final manuscript.

Acknowledgements We are grateful to Poliane Alfenas-Zerbini (Universidade Federal de Viçosa - UFV) for providing the polyclonal antibody against SITCTP. M. de Carvalho was recipient of a Ph.D. fellowship from FAPESP (2011/10309-3). L. M. de Araújo and M. L. C. Arcuri were recipients of undergraduate FAPESP fellowships (2011/17462-1 and 2014/03241-1). M. L. Acencio was a recipient of a PNPD/CAPES postdoctoral fellowship. I. G. Maia is recipient of a research fellowship from $\mathrm{CNPq}$.

\section{Compliance with ethical standards}

Conflict of interest The authors declare that they have no conflict of interest.

\section{References}

Alfenas-Zerbini P, Maia IG, Fávaro RD, Cascardo JC, Brommonschenkel SH, Zerbini FM (2009) Genome-wide analysis of differentially expressed genes during the early stages of tomato infection by a potyvirus. Mol Plant Microbe Interact 22:352-361

Anderson JP, Badruzsaufari E, Schenk PM, Manners JM, Desmond OJ, Ehlert C, Maclean DJ, Ebert PR, Kazan K (2004) Antagonistic interaction between abscisic acid and jasmonate-ethylene signaling pathways modulates defense gene expression and disease resistance in Arabidopsis. Plant Cell 16:3460-3479
Babitha KC, Vemanna RS, Nataraja KN, Udayakumar M (2015) Overexpression of EcbHLH57 transcription factor from Eleusine coracana $\mathrm{L}$. in tobacco confers tolerance to salt, oxidative and drought stress. PLoS One 10:e0137098

Begcy K, Mariano ED, Mattiello L, Nunes AV, Mazzafera P, Maia IG, Menossi M (2011) An Arabidopsis mitochondrial uncoupling protein confers tolerance to drought and salt stress in transgenic tobacco plants. PLoS One 6:e23776

Berkowitz O, Jost R, Pollmann S, Masle J (2008) Characterization of TCTP, the translationally controlled tumor protein, from Arabidopsis thaliana. Plant Cell 20:3430-3447

Bi Z, Merl-Pham J, Uehlein N, Zimmer I, Mühlhans S, Aichler M, Walch AK, Kaldenhoff R, Palme K, Schnitzler JP, Block K (2015) RNAi-mediated downregulation of poplar plasma membrane intrinsic proteins (PIPs) changes plasma membrane proteome composition and affects leaf physiology. J Proteomics 128:321-332

Bommer UA, Thiele BJ (2004) The translationally controlled tumour protein (TCTP). Int J Biochem Cell Biol 36:379-385

Brandalise M, Maia IG, Borecký J, Vercesi AE, Arruda P (2003) Overexpression of plant uncoupling mitochondrial protein in transgenic tobacco increases tolerance to oxidative stress. J Bioenerg Biomembr 35:203-209

Brioudes F, Thierry AM, Chambrier P, Mollereau B, Bendahmane M (2010) Translationally controlled tumor protein is a conserved mitotic growth integrator in animals and plants. Proc Natl Acad Sci USA 107:16384-16389

Bruckner FP, da Silva Xavier A, de Souza Cascardo R, Otoni WC, Zerbini FM, Alfenas-Zerbini P (2016) Translationally controlled tumor protein (TCTP) from tomato and Nicotiana benthamiana is necessary for successful infection by a potyvirus. Mol Plant Pathol. doi: $10.1111 / \mathrm{mpp} .12426$

Bueso E, Alejandro S, Carbonell P, Perez-Amador MA, Fayos J, Bellés JM, Rodriguez PL, Serrano R (2007) The lithium tolerance of the Arabidopsis cat 2 mutant reveals a cross-talk between oxidative stress and ethylene. Plant J 52:1052-1065

Cao WH, Liu J, Zhou QY, Cao YR, Zheng SF, Du BX, Zhang JS, Chen SY (2006) Expression of tobacco ethylene receptor NTHK1 alters plant responses to salt stress. Plant Cell Environ 29:1210-1219

Cao WH, Liu J, He XJ, Mu RL, Zhou HL, Chen SY, Zhang JS (2007) Modulation of ethylene responses affects plant salt-stress responses. Plant Physiol 143:707-719

Chaves MM, Flexas J, Pinheiro C (2009) Photosynthesis under drought and salt stress: regulation mechanisms from whole plant to cell. Ann Bot 103:551-560

Chen L, Ren F, Zhou L, Wang Q-Q, Zhong H, Li X-B (2012) The Brassica napus Calcineurin B-Like 1/CBL-interacting protein kinase 6 (CBL1/CIPK6) component is involved in the plant response to abiotic stress and ABA signalling. J Exp Bot 63:6211-6222

Chen Y, Chen X, Wang H, Bao Y, Zhang W (2014) Examination of the leaf proteome during flooding stress and the induction of programmed cell death in maize. Proteome Sci 12:33

Ermolayev V, Weschke W, Manteuffel R (2003) Comparison of Alinduced gene expression in sensitive and tolerant soybean cultivars. J Exp Bot 54:2745-2756

Falda M, Toppo S, Pescarolo A, Lavezzo E, Di Camillo B, Facchinetti A, Cilia E, Velasco R, Fontana P (2012) Argot2: a large scale function prediction tool relying on semantic similarity of weighted Gene Ontology terms. BMC Bioinform 13(Suppl 4):S14

Fontana P, Cestaro A, Velasco R, Formentin E, Toppo S (2009) Rapid annotation of anonymous sequences from genome projects using semantic similarities and a weighting scheme in gene ontology. PLoS One 4:e4619 
Ghabooli M, Khatabi B, Ahmadi FS, Sepehri M, Mirzaei M, Amirkhani A, Jorrín-Novo JV, Salekdeh GH (2013) Proteomics study reveals the molecular mechanisms underlying water stress tolerance induced by Piriformospora indica in barley. J Proteomics 94:289-301

Grabherr MG, Haas BJ, Yassour M, Levin JZ, Thompson DA, Amit I, Adiconis X, Fan L, Raychowdhury R, Zeng Q, Chen Z, Mauceli E, Hacohen N, Gnirke A, Rhind N, di Palma F, Birren BW, Nusbaum C, Lindblad-Toh K, Friedman N, Regev A (2011) Fulllength transcriptome assembly from RNA-Seq data without a reference genome. Nat Biotechnol 29:644-652

Grondin A, Rodrigues O, Verdoucq L, Merlot S, Leonhardt N, Maurel C (2015) Aquaporins contribute to ABA-triggered stomatal closure through OST1-mediated phosphorylation. Plant Cell 27:1945-1954

Gutiérrez-Galeano DF, Toscano-Morales R, Calderón-Pérez B, Xoconostle-Cázares B, Ruiz-Medrano R (2104) Structural divergence of plant TCTPs. Front Plant Sci 5:361

Hoepflinger MC, Reitsamer J, Geretschlaeger AM, Mehlmer N, Tenhaken R (2013) The effect of translationally controlled tumour protein (TCTP) on programmed cell death in plants. BMC Plant Biol 13:135

Horsch RB, Fry JE, Hoffmann NL, Eichholtz D, Rogers SG, Fraley RT (1985) A simple and general method for transferring genes into plants. Science 227:1229-1231

Kim YM, Han YJ, Hwang OJ, Lee SS, Shin AY, Kim SY, Kim JI (2012) Overexpression of Arabidopsis translationally controlled tumor protein gene AtTCTP enhances drought tolerance with rapid ABA-induced stomatal closure. Mol Cells 33:617-626

Kong L, Zhang Y, Ye ZQ, Liu XQ, Zhao SQ, Wei L, Gao G (2007) CPC: assess the protein-coding potential of transcripts using sequence features and support vector machine. Nucleic Acids Res 35(Suppl 2):W345-W349

Laitz AV, Acencio ML, Budzinski IG, Labate MT, Lemke N, Ribolla PE, Maia IG (2015) Transcriptome response signatures associated with the overexpression of a mitochondrial uncoupling protein (AtUCP1) in tobacco. PLoS One 10:e0130744

Li B, Dewey CN (2011) RSEM: accurate transcript quantification from RNA-Seq data with or without a reference genome. BMC Bioinform 12:323

Li D, Deng Z, Liu X, Qin B (2013) Molecular cloning, expression profiles and characterization of a novel translationally controlled tumor protein in rubber tree (Hevea brasiliensis). J Plant Physiol 170:497-504

Lin Z, Arciga-Reyes L, Zhong S, Alexander L, Hackett R, Wilson I, Grierson D (2008) SITPR1, a tomato tetratricopeptide repeat protein, interacts with the ethylene receptors NR and LeETR1, modulating ethylene and auxin responses and development. J Exp Bot 59:4271-4287

Maere S, Heymans K, Kuiper M (2005) BiNGO: a Cytoscape plugin to assess overrepresentation of gene ontology categories in biological networks. Bioinformatics 21:3448-3449

Maurel C, Boursiac Y, Luu DT, Santoni V, Shahzad Z, Verdoucq L (2015) Aquaporins in plants. Physiol Rev 95:1321-1358

Moradi F, Ismail AM (2007) Responses of photosynthesis, chlorophyll fluorescence and ROS-Scavenging systems to salt stress during seedling and reproductive stages in rice. Ann Bot 99:1161-1173

Murashige T, Skoog F (1962) A revised medium for rapid growth and bio-assays with tobacco tissue cultures. Physiol Plant 15:473-497

Nagano-Ito M, Ichikawa S (2012) Biological effects of mammalian translationally controlled tumor protein (TCTP) on cell death, proliferation, and tumorigenesis. Biochem Res Int 2012:204960

Prasch CM, Ott KV, Bauer H, Ache P, Hedrich R, Sonnewald U (2015) $\beta$-amylase1 mutant Arabidopsis plants show improved drought tolerance due to reduced starch breakdown in guard cells. J Exp Bot 66:6059-6067

Radivojac P, Clark WT, Oron TR et al (2013) A large-scale evaluation of computational protein function prediction. Nat Methods 10:221-227

Robinson MD, McCarthy DJ, Smyth GK (2010) edgeR: a Bioconductor package for differential expression analysis of digital gene expression data. Bioinformatics 26:139-140

Santa Brígida AB, dos Reis SP, Costa Cde N, Cardoso CM, Lima AM, de Souza CR (2014) Molecular cloning and characterization of a cassava translationally controlled tumor protein gene potentially related to salt stress response. Mol Biol Rep 41:1787-1797

Tao JJ, Cao YR, Chen HW, Wei W, Li QT, Ma B, Zhang WK, Chen SY, Zhang JS (2015) Tobacco translationally controlled tumor protein interacts with ethylene receptor tobacco histidine kinase 1 and enhances plant growth through promotion of cell proliferation. Plant Physiol 169:96-114

Toscano-Morales R, Xoconostle-Cázares B, Martínez-Navarro AC, Ruiz-Medrano R (2014) Long distance movement of an arabidopsis translationally controlled tumor protein (AtTCTP2) mRNA and protein in tobacco. Front Plant Sci 5:705

Toscano-Morales R, Xoconostle-Cázares B, Cabrera-Ponce JL, Hinojosa-Moya J, Ruiz-Salas JL, Galván-Gordillo SV, GuevaraGonzález RG, Ruiz-Medrano R (2015) AtTCTP2, an Arabidopsis thaliana homolog of translationally controlled tumor protein, enhances in vitro plant regeneration. Front Plant Sci 6:468

Upchurch RG (2008) Fatty acid unsaturation, mobilization, and regulation in the response of plants to stress. Biotechnol Lett 30:967-977

Valerio C, Costa A, Marri L, Issakidis-Bourguet E, Pupillo P, Trost P, Sparla F (2011) Thioredoxin-regulated beta-amylase (BAM1) triggers diurnal starch degradation in guard cells, and in mesophyll cells under osmotic stress. J Exp Bot 62:545-555

Wang ZQ, Li GZ, Gong QQ, Li GX, Zheng SJ (2015) OsTCTP, encoding a translationally controlled tumor protein, plays an important role in mercury tolerance in rice. BMC Plant Biol $15: 123$

Yao X, Xiong W, Ye T, Wu Y (2012) Overexpression of the aspartic protease ASPG1 gene confers drought avoidance in Arabidopsis. J Exp Bot 63:2579-2593

Zhang M, Barg R, Yin M, Gueta-Dahan Y, Leikin-Frenkel A, Salts Y, Shabtai S, Ben-Hayyim G (2005) Modulated fatty acid desaturation via overexpression of two distinct omega-3 desaturases differentially alters tolerance to various abiotic stresses in transgenic tobacco cells and plants. Plant J 44:361-371 